\title{
Changement organisationnel : Quel rôle pour les
} acteurs ?

\section{Organizational change: What role for the actors?}

\author{
Fatima Ezzahra NOUIKER \\ Laboratoire : Gestion des compétences, de l'innovation et des aspects sociaux des organisations \\ (GECIAS) \\ Faculté des sciences juridiques, économiques et sociales \\ Université Hassan II - Casablanca - Maroc
}

\begin{abstract}
Résumé : De nos jours, le changement des organisations aussi bien privées que publiques s'impose pour affronter un environnement caractérisé par l'évolution rapide et accélérée. Il peut avoir pour objectifs le développement de l'organisation, la réalisation de la performance, la résolution d'un problème ou la survie tout simplement en cas de risque de disparition du marché ...

A ce niveau, le changement ne peut se réaliser sans la présence d'un certain nombre d'acteurs ayant chacun un rôle à jouer pour faire aboutir le processus. Il s'agit du leader de l'organisation, du chef de projet, de l'équipe de changement, des managers intermédiaires, des destinataires du changement et des consultants externes.

Le présent article a pour objectif d'expliciter les rôles des acteurs du changement à partir de la revue de littérature y relative.
\end{abstract}

Mots-clés : Changement organisationnel; acteurs du changement organisationnel; leader du changement ; chef de projet ; managers intermédiaires.

Digital Object Identifier (DOI): https://doi.org/10.52502/ijesm.v1i1.148 


\section{Introduction}

Dans le monde des organisations, le changement n'est plus un mot étranger. Toutes les organisations aussi bien privées que publiques, optent pour des changements à un moment ou à un autre de leur vie. Ceci d'ailleurs a suscité l'intérêt des théoriciens ce qui justifie la richesse de la littérature en la matière.

En effet, le changement est évoqué par plusieurs disciplines dont la théorie de développement organisationnel, la théorie de contingence, le management stratégique ... Chacune de ces disciplines apporte sa vision du concept, ce qui permet d'aborder les différentes dimensions qui l'entourent. Les premiers écrits datent des années soixante.

Les acteurs constituent, à ce titre, une composante très importante pour conduire le changement. Ce sont des ressources clés pour son implémentation, chacun ayant un rôle à jouer dans le processus. Il s'agit du leader de l'organisation, du chef de projet, de l'équipe de changement, des managers intermédiaires, des destinataires du changement et des consultants externes. Les managers intermédiaires et les destinataires sont les acteurs qui peuvent manifester le plus de résistance étant donné que les autres sont plus impliqués dans le processus de changement. Les leaders sont souvent ceux qui initient ce dernier. A noter que les managers intermédiaires ont une position critique et compliquée lors du changement du fait qu'ils sont à la fois des destinataires et des transmetteurs de changement. Ils doivent motiver l'équipe pour changer et changer personnellement en plus de l'activité courante. Il leur faut ainsi de l'accompagnement, du suivi et de la formation dans ce sens ...

Dans ce qui suit, une présentation détaillée des rôles de ces acteurs à partir de la revue de littérature, après une présentation du concept du changement organisationnel en général.

\section{Changement organisationnel}

Le changement organisationnel a plusieurs définitions dans la littérature. Le mot changer vient du latin tardif « cambiare », ayant le sens de « troquer, rendre autre, modifier» (CNRTL, 2009) ${ }^{1}$. Le terme est associé à d'autres mots tels devenir, s'ajuster, s'adapter, transformer, évoluer, se modifier (Beaudoin, 1990 ; Grouard et Meston, 1998). Le changement consiste selon Faurie (2008), à faire autre chose avec les mêmes moyens.

D'après Poole et Van de Ven (2004), il s'agit d'une différence de forme, qualité ou état d'une entité organisationnelle dans le temps. Le changement est déclenché par un événement discret permettant de passer d'un état non-congelé à un état gelé (Van de Ven et al., 1999). Il consiste en un ensemble de séquences imbriquées d'événements qui se déroulent dans le temps au niveau du développement des individus, des organisations et des industries (Garud et Van De Ven, 2006).

Le changement est justifié par l'inadéquation de l'état ou de la situation originale, visant à se modifier vers un état désiré, jugé plus adapté et répondant mieux aux exigences du milieu ou aux nouvelles aspirations des personnes concernées (Bélanger, 1994). Il se concrétise selon Autissier et al. (2010) par la perte d'un existant connu pour un avenir justifié par un progrès.

Le changement organisationnel se défini également entant que processus (Grouard et Meston, 1998 ; Guilhon, 1998 ; Nurcan et al., 2002) de transformation radicale ou marginale (Grouard et Meston, 1998 ; Guilhon, 1998). Ce changement peut concerner les structures et les compétences d'une organisation (Grouard et Meston, 1998 ; Guilhon, 1998), couvrir un sous-système (Collerette et al., 1997), et toucher

\footnotetext{
${ }^{1}$ Centre National des Ressources Textuelles et Lexicales
} 
une composante du cadre organisationnel (structure, culture, procédures, technologies, conditions de travail, modalités de management...) (Bec et al., 2007).

Le processus est l'une des parties composant le changement organisationnel selon Pettigrew (1987). En effet, d'après cet auteur, le changement se décompose en trois parties : le contenu, le contexte et le processus. Le contenu vise d'apporter une réponse à la question du « quoi » du changement (Walker et al., 2007), il renvoi aux objectifs et missions de celui-ci. Le contexte désigne l'environnement interne et externe de l'organisation (Walker et al., 2007) ainsi que les acteurs concernés par le changement. Le processus est considéré entant qu'un ensemble d'actions et d'évènements continus et interdépendants utilisés pour expliquer les origines, la continuité et les résultats des phénomènes observés (Pettigrew, 1987).

En somme, on peut conclure que le changement organisationnel désigne le passage d'un état présent à un état futur (Collerette et al., 1997 ; Bélanger, 1994 ; Hafsi et Fabi, 1997 ; Nurcan et al., 2002 ; Autissier et al., 2010), observé dans l'environnement et perceptible par les acteurs, les membres ou les personnes qui sont en relation avec le système en question ou dont le changement introduit doit modifier les habitudes de travail (Collerette et al., 1997 ; Do Espirito, 2006). Ce changement est repérable dans le temps (Poole et Van de Ven, 2004 ; Do Espirito, 2006), et a un caractère relativement durable (Collerette et al., 1997; Do Espirito, 2006).

Le concept de changement traverse les travaux des différentes disciplines de management, apportant chacune une réponse différenciée à la problématique. Autissier et al. (2010) présente chronologiquement l'évolution du concept en mentionnant l'apport de chaque discipline au sujet.

Ainsi, dès les années soixante, le management stratégique a apporté une réponse à la problématique via la planification adoptée par les dirigeants en vue de s'adapter aux exigences du marché (Autissier et al., 2010). A la même époque, émerge la théorie de contingence (Burns et Stalker, 1961 ; Chandler, 1989 ; Woodward, 1965 cités dans Autissier et al., 2010). Elle s'articule autour des raisons motivant le dirigeant à maîtriser l'évolution de son organisation et à améliorer les performances de celle-ci en s'adaptant à l'environnement. Or, cette théorie fonctionne dans des sphères caractérisées par moins de turbulence ou en croissance (Autissier et al., 2010). Ainsi, la théorie de la contingence a bien démontré ses limites dès les années soixante-dix marquées par une instabilité de l'environnement des affaires (Autissier et al., 2010).

Ces contextes instables marqués par une libération des marchés, une concurrence forte, une réduction des coûts et une compétitivité accrue ont exigé un changement radical des stratégies et des cultures organisationnelles et ont nécessité le développement des capacités d'anticipation des évolutions futures de l'environnement pour pouvoir s'y adapter. Ce type de changement à risque élevé est justifié par une situation de crise et est initié par le dirigeant capable de mobiliser l'ensemble des acteurs en relation avec le projet et de maîtriser le processus de changement (Autissier et al., 2010).

Ces théories et courants de pensée ont démontré leurs limites au début des années quatre-vingt suite à l'émergence d'environnements de plus en plus instables et imprévisibles. Ainsi, des auteurs tels Mintzberg et Waters (1985) ont montré que la stratégie n'est pas uniquement le résultat d'intentions et de planification initiale mais aussi de stratégies émergentes susceptibles de modifier les objectifs de départ (cités dans Autissier et al., 2010). Egalement, d'autres auteurs tels Quinn (1980) et Smith (1986) ont privilégié le changement progressif à partir d'ajustements menant à la transformation globale de l'organisation en critiquant le changement radical (cités dans Autissier et al., 2010).

Ces facteurs ont poussé les organisations à vouloir se munir de la capacité de l'auto-transformation en maîtrisant les pratiques systématiques et l'innovation permettant l'amélioration continue ou le développement de nouvelles activités (Autissier et al., 2010). 
Cette volonté de maîtriser le changement vient de sa nécessité pour les organisations d'aujourd'hui. Selon plusieurs dirigeants et responsables, le changement se présente comme la solution à la gestion de toutes les évolutions qu'une organisation doit intégrer pour survivre et se développer (Autissier et al., 2010). Il constitue un enjeu managérial, une obligation et une préoccupation prioritaire pour l'ensemble des responsables (Autissier et al., 2010 ; Bec et al., 2007).

Selon la littérature portant sur le sujet, la mise en place du changement organisationnel est motivée par plusieurs objectifs qui justifient sa légitimité. Le changement vise entre autres le développement de l'organisation (Autissier et al., 2010 ; Balogun et Hailey, 2005). C'est un moyen pour réaliser la croissance, l'évolution, le progrès ou dans une moindre mesure la survie (Autissier et al., 2010). Il permet de s'adapter aux changements de l'environnement (Autissier et al., 2010 ; Bec et al., 2007), ainsi qu'aux évolutions technologiques (Balogun et Hailey, 2005 ; Bec et al., 2007 ; Do Espirito, 2006). Le changement se présente aussi comme un dispositif permettant l'efficacité et l'efficience. En effet, il se met en place dans l'objectif de réduire les coûts et d'améliorer la productivité (Bec et al., 2007). Il vise également de mieux répondre aux besoins et demandes des usagers (Bec et al., 2007 ; Do Espirito, 2006), améliorer la qualité du service rendu (Bec et al., 2007) et élever la performance en adoptant une organisation flexible et des manières de travail plus globalement intégrées (Balogun, 2007). Le changement offre aussi une opportunité d'apprentissage pour les individus et l'organisation (Autissier et al., 2010) et permet la consolidation de certains secteurs en cas de fusions et d'acquisitions (Balogun et Hailey, 2005).

Durant ces vingt dernières années, le changement s'insère dans ce qu'on appelle les effets de mode à travers l'externalisation (Balogun et Hailey, 2005), la gestion de la qualité totale et la recherche de compétitivité (Balogun et Hailey, 2005 ; Bec et al., 2007).

En bref, le monde des organisations qu'elles soient de statut privé ou public vit, à nos jours, sous la pression des modernisations, des réformes et des restructurations... En effet, les objectifs de réactivité, de flexibilité, d'adaptabilité, de mobilité... poussent de plus en plus les organisations à changer (Bec et al., 2007). Ces dernières se transforment, se complexifient et évoluent. Donc une vision linéaire du monde serait inadaptée à la complexité de l'organisation d'aujourd'hui (Faurie, 2008), d'où la nécessité du changement.

\section{Acteurs du changement organisationnel}

La mise en place du changement organisationnel nécessite la présence de plus qu'un individu. Elle ne peut être assurée par une seule personne, le porteur ou le chef de projet mais le soutien d'autres acteurs est nécessaire notamment dans le cas de changement stratégique vue son niveau de complexité et la multiplicité des tâches devant être conduites (Balogun et Hailey, 2005). Ainsi, de nos jours, la présence d'une personnalité charismatique et héroïque n'est plus suffisante pour mener à bien un changement (Balogun et Hailey, 2005).

Cette partie présente les différents acteurs du changement et le rôle qu'ils peuvent jouer.

\subsection{Rôle du leader de l'organisation}

Selon Balogun et Hailey (2005), le leadership revêt une grande importance pour mettre en œuvre des transformations de grande ampleur incarné par un champion engagé et enthousiaste pour appliquer sa vision.

Le leader, une figure clé et centrale de l'organisation, constitue un acteur crucial pour la réussite du changement. Au niveau de l'entreprise, le « leader » peut être le PDG, le directeur général, le directeur des ressources humaines ou autre directeur, et même un cadre supérieur. Les organisations multinationales peuvent comprendre plusieurs leaders répartis sur les différentes composantes de 
l'entreprise sous la responsabilité d'un leader central (Balogun et Hailey, 2005). Dans le secteur public à l'instar d'un ministère par exemple, le leader peut être le ministre, le secrétaire général, un directeur ou même un manager intermédiaire ou de proximité pour les changements de petite ampleur.

Le leader susceptible de se représenter entant qu'une direction permet la création d'un élan, d'une détermination et d'une grande focalisation sur le projet, objet de changement. Toutefois, le leader peut ne pas disposer d'assez de temps en ayant d'autres occupations professionnelles plus prioritaires (Balogun et Hailey, 2005).

Le leader conçoit les orientations stratégiques et plans et supervise les actions de celui chargé de la mise en œuvre (Gioia et Chittipeddi, 1991).

\subsection{Rôle du porteur de changement}

Le porteur de changement ou le chef de projet est la personne qui mène et conduit le changement (Bec et al., 2007). En effet, la mise en œuvre du changement ne peut avoir lieu que si quelqu'un en est responsable même si cette responsabilité peut être partagée entre plusieurs personnes (Balogun et Hailey, 2005). Ce rôle peut être accompli par différents acteurs : directeur général, directeur des ressources humaines, une équipe de quelques personnes... (Balogun et Hailey, 2005).

Pour bien mener le changement, le chef de projet doit avoir certaines qualités qui d'après Balogun et Hailey (2005) sont de trois ordres : deux qualités de gestion (capacité d'analyse et capacité de mise en œuvre) et une qualité personnelle (conscience de soi).

La compétence de la capacité d'analyse permet au chef de projet de disposer d'une image globale de l'organisation, lieu du changement, de saisir sa culture et de comprendre les motivations de son personnel à condition de savoir prioriser les traits les plus importants vis-à-vis de la situation en vue de décider de ceux critiques au succès du changement.

La capacité à gérer la mise en œuvre se réfère à l'action pratique concernant le changement tout en déterminant le type d'interventions à réaliser et l'ordre de réalisation.

La conscience de soi désigne l'aptitude de l'individu à comprendre ses propres préjugés, préférences et expériences, pour minimiser le risque que sa perception des choses impacte l'approche de changement choisie et les interventions effectuées en négligeant le contexte réel de changement (Balogun et Hailey, 2005).

Etre conscient de soi c'est aussi savoir différencier les orientations objectives et subjectives (Balogun et Hailey, 2005). Pour les orientations objectives, les décisions prises sont basées sur des données tangibles et chiffrées et les jugements sont concrets et mesurables. Les orientations subjectives se caractérisent par des jugements fondés sur des faits immatériels, intuitifs et qualitatifs.

Le manager vigilent choisit la méthode adaptée à la situation de changement en prenant de la distance par rapport à ses préférences et orientations (Balogun et Hailey, 2005).

D'autres qualités sont importantes pour bien conduire le changement. Il s'agit entre autres de la capacité de gérer la complexité et d'avoir un pouvoir d'influence sur les acteurs cibles (Balogun et Hailey, 2005).

Le chef de projet doit être capable de mettre en œuvre les plans de changement, d'anticiper, d'identifier et de gérer l'imprévu et de mettre en cohérence les programmes formulés et leur implémentation, de superviser l'ensemble des modifications apportées et de contrôler l'avancement du projet (Balogun et Hailey, 2005).

Le chef de projet peut gérer tout seul la transition, toutefois, cela nécessite du temps, notamment pour les grandes transformations, d'où l'intérêt d'avoir une équipe dédiée à cette tâche disposant de 
compétences multidisciplinaires nécessaires à l'accomplissement du projet (Balogun et Hailey, 2005). En plus des compétences techniques, il existe des compétences managériales tels ; la communication, le coaching, la capacité à créer l'esprit d'équipe, la diplomatie et la capacité à motiver le personnel et les compétences interpersonnelles relatives au contrôle, à la planification, à la budgétisation et à la gestion de projets ou à la maîtrise des systèmes d'information (Balogun et Hailey, 2005).

\subsection{L'équipe du changement}

La transformation peut être menée par une équipe de changement ou une équipe projet qui a besoin du soutien des dirigeants si elle ne dispose pas d'acteurs influents et puissants notamment en ce qui concerne les transformations de grande ampleur (Balogun et Hailey, 2005). La désignation d'une équipe de changement a un certain nombre d'avantages tels ; l'appropriation des initiatives de changement, la connaissance de l'organisation et des enjeux... et éventuellement la persévérance et le suivi en cas de présence d'individus potentiellement influents dans l'équipe de changement (Balogun et Hailey, 2005). Toutefois, la conception du changement peut consommer du temps ce qui exige des compromis entre les membres de l'équipe et cette dernière peut manquer de pouvoir pour mener à bien le changement (Balogun et Hailey, 2005).

\subsection{Les managers intermédiaires}

Les managers intermédiaires ou les cadres moyens sont des acteurs critiques au changement. Alors qu'ils sont vus par certains comme des personnes résistantes au changement qui bloquent la réalisation des transformations ne servant pas leurs intérêts personnels, d'autres études stipulent que le contexte organisationnel est celui qui les empêche de mettre en œuvre les changements demandés par la direction (Balogun et Hailey, 2005).

Le rôle joué par les niveaux intermédiaires de la hiérarchie est important dans le cadre du changement (Balogun et Hailey, 2005). Ils sont aussi bien des objets que des acteurs de changement vue qu'ils sont souvent dans l'obligation de changer personnellement, de mettre en œuvre les changements exigés au niveau de leurs services ou équipes et d'aider et de guider leurs collaborateurs à évoluer sur le plan individuel tout en continuant à exercer leur activité courante (Balogun et Hailey, 2005).

L'intérêt que revêtissent les managers intermédiaires en plus des destinataires de changement dans le cadre des programmes de transformation se reflète aussi dans l'interprétation et le sens qu'ils construisent par rapport aux initiatives de changement lancées par les tops managers et qui influencent les outputs de celles-ci en produisant des résultats imprévus par rapport à ce qui était attendu (Balogun, 2006). Ces interprétations peuvent modifier les plans de départ initiés par les managers séniors, aussi elles sont plus influencées par les interactions informelles entre les gestionnaires intermédiaires c'est-àdire la communication latérale que par la communication verticale et les plans des managers séniors d'où la difficulté de prédire les résultats finaux (Balogun, 2006). Tous ces facteurs justifient l'intérêt pour les managers séniors de bien saisir le rôle que peuvent jouer les managers intermédiaires et la façon dont ils interprètent le changement et comment ceci peut influencer leur comportement, ainsi que de les impliquer dans le processus pour créer une idée partagée du changement et de donner eux même l'exemple en vivant le changement qu'ils veulent que les autres adoptent (Balogun, 2006).

En vue d'avoir un minimum de contrôle sur la communication informelle relative au processus de construction de sens du changement, les tops managers peuvent affecter aux managers intermédiaires des rôles précis et simples facilitant l'orientation de leurs actions et comportements dans le sens voulu en évitant qu'ils développent des rôles non planifiés pour défendre leurs propres intérêts ce qui peut entraver la réalisation des objectifs des projets de changement (Balogun, 2006). Alors, les managers intermédiaires ne doivent plus être considérés comme des récipiendaires passifs du changement mais 
des créateurs actifs de celui-ci, des traducteurs et des éditeurs de plans et de programmes de changements (Balogun, 2006).

La tâche des managers intermédiaires est plus compliquée dans le cadre des changements imposés où ils sont en même temps des destinataires que des metteurs en œuvre de plans qu'ils n'ont pas conçus (Balogun, 2007).

\subsection{Les récipiendaires du changement}

Les récipiendaires ou destinataires du changement sont des individus directement affectés par le changement et qui doivent s'y adapter (Balogun et Hailey, 2005). Ce processus peut être difficile nécessitant un soutien dans la gestion de la transition (Balogun et Hailey, 2005).

Certains collaborateurs, destinataires du changement, peuvent subir suite à un changement difficile qui impacte leurs conditions de travail des effets néfastes se traduisant à titre illustratif par une baisse de moral, du stress et des performances médiocres, susceptibles de perdurer en absence de traitement (Balogun et Hailey, 2005).

Les destinataires du changement comme les managers intermédiaires ne doivent pas être vus uniquement comme une source de résistance, leurs interprétations permettent de traduire les programmes de changement initiés par les managers séniors sur le terrain (Balogun, 2006).

En plus du facteur de la résistance, la justice organisationnelle constitue un autre élément auquel s'intéressent les destinataires du changement (Balogun et Hailey, 2005). Les collaborateurs jugent ce qui leur arrive comme juste et équitable en se comparant aux autres (Balogun et Hailey, 2005).

Dans cette optique, la prise en compte et la gestion du facteur émotionnel dans le cadre du changement est cruciale pour éviter des réactions contreproductives (Balogun et Hailey, 2005). La communication ouverte et l'implication des collaborateurs dans le processus peuvent être bénéfiques sur ce point visant le renforcement de l'esprit d'équipe (team building), de la confiance, du moral et de l'engagement du personnel, la facilitation du changement individuel via le conseil et le coaching, la diminution du sentiment d'insécurité relatif au poste et à la carrière via la gestion du stress (Balogun et Hailey, 2005).

Ces actions peuvent s'intégrer dans le cadre d'une approche globale de conduite du changement permettant d'influencer les réactions des récipiendaires de changement dans le sens désiré. Elle consiste en l'adoption d'une politique de proximité aux destinataires en vue de bien saisir leurs interprétations vis-à-vis du changement (Balogun, 2006).

Comme les managers intermédiaires, les interprétations des destinataires du changement sont construites suite à un processus de sensemaking plus influencé par la communication latérale que par celle verticale et les plans des managers séniors (Balogun, 2006). Ce processus influence fortement les résultats du changement et doit par conséquent être réfléchi dans le cadre des plans conçus par les leaders (Balogun, 2006). Ce processus informel peut être géré par les managers en s'y impliquant par la désignation de représentants qui participent dans les échanges, histoires, gossips... donnant l'exemple par leurs actions (Balogun, 2006). L'organisation des manifestations qui rassemblent le personnel pour discuter leurs idées peut être aussi utile (Balogun, 2006).

En somme, la communication via toutes ses images couvre une importance capitale dans l'alignement des interprétations à condition de saisir le pourquoi et le comment de ces interprétations (Balogun, 2006).

\subsection{Les consultants externes}

Plusieurs organisations publiques ou privées font appel à des cabinets et expertises externes pour mener les différents projets de changement. 
Ainsi, selon Balogun et Hailey (2005), les facilitateurs ou consultants externes peuvent avoir un apport important au processus de changement, et ce de différentes manières : conseils, formation en conduite de changement et même participation à la réalisation des projets de changement (Balogun et Hailey, 2005).

Le recours à des consultants externes a aussi bien des avantages que des inconvénients. Les avantages s'incarnent dans l'expérience des consultants, leur dévouement au projet, l'objectivité, la possibilité de servir de boucs émissaires pour annoncer les mauvaises nouvelles, la promotion des échanges sur la nécessité du changement et la capacité de neutraliser les barrières organisationnelles (Balogun et Hailey, 2005). Toutefois, le coût des consultations peut être très élevé (Balogun et Hailey, 2005). Egalement, les consultants n'ont pas de responsabilité à assumer ni d'appropriation, ni besoin de résultat et peuvent ne pas avoir suffisamment de connaissances sur l'activité de l'organisation (Balogun et Hailey, 2005).

\section{Conclusion}

Le besoin et le nombre des acteurs impliqués dans le changement dépendent de la nature et de l'ampleur de celui-ci. Chacun, comme précité, joue un rôle dans la mise en place des transformations.

Le leader du changement est une figure clé pour la réalisation de ce dernier, notamment en cas de projets de grande envergure.

Les changements ont besoin également pour leur mise en place d'un chef de projet qui soit responsable de leur réalisation. Ils peuvent aussi exiger la constitution d'une équipe de changement ou de projet qui contribue à leur mise en place.

Les managers intermédiaires sont d'une très grande importance vue qu'ils sont aussi bien des récipiendaires que des transmetteurs de changement vis-à-vis de leurs collaborateurs.

Les destinataires visés par le changement sont des individus affectés par celui-ci et qui doivent être impliqués pour permettre une exploitation des résultats.

A noter que plusieurs projets sont externalisés aujourd'hui pour tirer profit de l'expertise des consultants externes qui peuvent jouer un rôle important dans l'aboutissement des projets.

\section{BIBLIOGRAPHIE}

[1] A. Guilhon, « Le changement organisationnel est un apprentissage, » Revue française de gestion, $\mathrm{N}^{\circ} 120$, pp. 98-107, 1998.

[2] A.H. Van de Ven, D. Polley, R. Garud, and S. Venkataraman, The innovation journey, New York: Oxford University Press, 1999.

[3] A.M. Pettigrew, The Management of Strategic Change, Oxford, Basil Blackwell, 1987.

[4] B. Grouard, and F. Meston, L'entreprise en mouvement : Conduire et réussir le changement, Editions Dunod, 1998.

[5] C. Faurie, Conduire le changement : transformer les organisations sans bouleverser les hommes, L'harmattan, 2008.

[6] D. Autissier, I. Vandangeon-Derumez, and A. Vas, Conduite du changement : concepts-clés, Editions Dunod, 2010.

[7] D. Gioia, and K. Chittipeddi, "Sensemaking and sensegiving in strategic change initiation," Strategic Management Journal, vol. 12, $\mathrm{N}^{\circ}$ 6, pp. 433-448, 1991 
[8] H.J. Walker, A.A. Armenakis, and J.B. Bernerth, "Factors influencing organizational change efforts - An integrative investigation of change content, context, process and individual differences," Journal of Organizational Change Management, vol. 20, Nº, pp. 761-773, 2007.

[9] J. Balogun, "Managing Change: Steering a Course between Intended Strategies and Unanticipated Outcomes,” Long Range Planning, vol. 39, N (1), pp. 29-49, 2006.

[10] J. Balogun, “The Practice of Organizational Restructuring: From Design to Reality,” European Management Journal, vol. 25, $\mathrm{N}^{\circ}$ (2), pp. 81-91, 2007

[11] J. Balogun, V. H. Hailey, and E. Viardot, Stratégies du changement, 2d ed., Pearson, 2005.

[12] J. Bec, F. Granier, and J. Singér, La facilitation des changements organisationnels, Editions Harmattan, 2007.

[13] L. Bélanger, "Le changement organisationnel et le développement, » in La dimension humaine des organisations, N. Côté, L. Bélanger, and J. Jacques, Eds. Gaëtan Morin, 1994, pp 357-386

[14] M.S. Poole, and A.H Van de Ven, Handbook of Organizational Change and Innovation, Oxford University Press, 2004.

[15] P. Beaudoin, La Gestion du changement : Une approche Stratégique pour l'entreprise en mutation, Stratégies d'entreprise, 1990

[16] P. Collerette, G. Delisle, and R. Perron, Le changement organisationnel : théorie et pratique, Presse de l'Université du Québec, 1997.

[17] R. Garud, and A.H. Van de Ven, "Strategic Change Process," in Handbook of Strategy and Management, A. Pettigrew, and H. T. Whittington, Eds. London, UK: Sage, 2006, pp. 206-231.

[18] S. Nurcan, J. Barrios, and C. Rolland, "Une méthode pour la définition de l'impact organisationnel du changement, » Revue des Sciences et Technologies de l'Information - Série ISI : Ingénierie des Systèmes d'Information, Lavoisier, 2002

[19] T. Do Espirito, La communication du changement : les clés pour réussir la transformation de son entreprise, CFPJ Editions, 2006.

[20] T. Hafsi, and B. Fabi, Les fondements du changement stratégique, Les éditions Transcontinental, 1997. 